\title{
Utilização de Ensaios Não Destrutivos, Ultrassom e Pacômetro, como Métodos de Verificação da Resistência e Qualidade do Concreto em um Edifício em Aracaju - Sergipe
}

Use of non-destructive testing, ultrasound and cover meter, as method for check the resistance and quality of concrete in a building in Aracaju - Sergipe

\section{Emanuella Carvalho ${ }^{1}$ (D) orcid.org/0000-0002-3945-4152}

Caroline Sena ${ }^{1}$ (D) orcid.org/0000-0003-3557-7056

Mikaele Rocha ${ }^{2}$ (D) orcid.org/0000-0002-9839-7813

Carlos Carvalho ${ }^{1}$ (D) orcid.org/0000-0002-3791-0812

${ }^{1}$ Departamento de Engenharia Civil, Instituto Federal de Ciência, Educação e Tecnologia de Sergipe, Aracaju, Brasil,

2 Programa de Pós-Graduação em Engenharia de Segurança do Trabalho, Faculdade de Administração e Negócios de Sergipe, Aracaju, Brasil.

E-mail do autor principal: Emanuella Carvalho emanuellaacarvalho@hotmail.com

\section{Resumo}

O concreto é um dos principais materiais utilizados na construção civil. No processo de verificação de sua qualidade de produção e resistência mecânica, o ensaio comumente realizado é o de compressão destrutiva axial em corpos-de-prova conforme prescrições da NBR 5739:2007. Porém, a possibilidade de monitorar essa qualidade e propriedades mecânicas recorrendo a ensaios não destrutivos (ENDs) facultaria a vantagem adicional dessas análises sem danos às estruturas e/ou corpos-de-prova, já na fase da produção. A portabilidade dos equipamentos nas medições em campo sem a necessidade de recorrer aos laboratórios especializados facilitaria sobremaneira esse monitoramento contínuo do controle de qualidade. Com esse objetivo, procedeu-se a um estudo de caso utilizando-se a pacometria e a ultrassonografia nos termos da NBR 8802/2013 e, com o auxílio do Software OriginPro e da expressão matemática do coeficiente de Pearson, analisou-se a existência de correlação aceitável estatisticamente entre os valores medidos das velocidades ultrassonoras com os resultados dos ensaios de compressão axial laboratoriais. Elaborou-se gráficos de dispersão que destacaram uma correlação aceitável e também, através da pacometria, inspecionou-se a qualidade de produção dos concretos estruturais executados. Ficou evidente a viabilidade dos ensaios não destrutivos nos canteiros de obras para o monitoramento contínuo do controle de qualidade estrutural.

Palavras-Chave: Resistência; Ensaios não destrutivos; Correlação; Controle de qualidade. 
Utilização de ensaios não destrutivos, ultrassom e pacômetro, como métodos de verificação da resistência e qualidade do concreto em um edifício em Aracaju - Sergipe

\begin{abstract}
The concrete is one of the main materials used in construction. In the process of checking its production quality and mechanical strength, the most commonly performed test for that is the destructive test of axial compression in specimens, following indications described in NBR 5739:2007. However, the possibility to check that quality and mechanical properties using non-destructive testing (NDTs) would provide an extra advantage of those analyses without damage to the structure and/or the specimen, already in the production stage. The equipment's portability in field measurements without the need to resort to specialized laboratories would severely facilitate this continuous tracking of the quality control. To this end, a case study using cover meter and ultrasound was performed according to NBR 8802/2013, and, with the aid of the Software OriginPro and of Pearson correlation coefficient, was analyzed the existence of acceptable statistically correlation between the measured values for ultrasonic speed and the axial compression test results done in laboratory. Scatter plots were elaborated and highlighted an acceptable correlation and, using cover meter, the production quality of structural concrete was inspected. It seemed clear the feasibility of non-destructive testing in the building sites to the continuous monitoring of the structural quality control.
\end{abstract}

Key-words: Resistance; Non-destructive testing; Correlation; Quality control. 


\section{Introdução}

A resistência à compressão é a propriedade do concreto adotada por ocasião do dimensionamento da estrutura. Portanto, está diretamente ligada à segurança e estabilidade estrutural. [1]

Além da resistência à compressão, a resistência mecânica e índice de vazios nos concretos são também propriedades fundamentais para o desempenho pleno da vida útil e durabilidade das estruturas.

Segundo Evangelista (2002) [2], comumente são feitos ensaios de resistência à compressão aos 28 (vinte e oito) dias em corpos-de-prova cilíndricos para verificar se o concreto está coerente com o especificado em projeto. Esses cilindros são retirados do mesmo concreto, ainda no estado fresco, e moldados no local da construção. São encaminhados para laboratório especializado em ensaios de resistência à compressão e outras propriedades. Porém, caso haja a necessidade de analisar a situação em obra acabada, a retirada dos corpos-de-prova provoca danos ao componente requerendo reparos localizados na estrutura.

Tem-se verificado uma vasta aplicação dos ensaios in situ em diversos países, assim como um grande número de pesquisas nessa área, visando a obtenção de resultados mais confiáveis nas investigações das propriedades do concreto das estruturas. [2]

Logo, existindo a possibilidade de se analisar a resistência mecânica e a qualidade de produção do concreto por meio de ensaios não destrutivos (ENDs), fomentar-se-iam ganhos financeiros e técnicos, em tempo e praticidade, tendo em vista que não haveria a necessidade de realizar reparos e os ensaios poderiam simultaneamente monitorar essas características durante e/ou logo após a sua execução.

Carvalho (2013) [3] cita que nas construtoras e nas usinas de concreto, o controle de qualidade do material e da estrutura como um produto necessita utilizar ENDs como ferramenta que permita análise rápida de verificação da resistência, homogeneidade e módulo de elasticidade do concreto.

Eventos científicos, trabalhos e teses têm surgido sobre o tema do uso dos ENDs na construção civil particularmente sobre estruturas de concreto. Enfatizando a importância imperiosa do seu uso na indústria da construção. [3]

30
Sendo assim, como citado por Schiavon (2015) [4], os ensaios não destrutivos visam avaliar um elemento sem gerar danos a ele com a técnica empregada. Um tipo de ensaio não destrutivo utilizado em concreto é o método da velocidade do pulso de ondas ultrassônicas.

O ensaio por ultrassom caracteriza-se num método não destrutivo que tem por objetivo a detecção de defeitos ou descontinuidades internas presentes nos mais variados tipos ou forma de materiais. [5]

Verificando a sua compacidade por análise das velocidades da onda ultrassônica obtida nos determinados elementos estruturais, no qual, quanto maior sua velocidade melhor produzido está o concreto, logo que menos tempo levará para a onda ser transmitida e recebida pelos dois transdutores do ultrassom, mostrando que a presença de vazios em seu interior é muito baixa ou nula, sabendo que, como cita Granato (2002) [6], a compacidade do concreto é a propriedade mais importante do mesmo nos efeitos de sua resistência à penetração dos agentes agressivo.

Os ensaios não destrutivos fornecem informações quanto às propriedades do concreto das estruturas, mas a confiabilidade da avaliação dessas propriedades depende da experiência do profissional que os realiza e do emprego de curvas de correlação entre as grandezas medidas nos ensaios com as propriedades conhecidas do concreto investigado. [7]

Assim sendo, para que os métodos de ensaio in situ sejam utilizados para avaliar a resistência do concreto, são necessárias curvas de correlação entre os resultados destes ensaios e a resistência à compressão do concreto. [2]

Dessa forma, o presente artigo teve como objetivo realizar ensaios de velocidade de propagação de ondas ultrassônicas, seguindo a ABNT NBR 8802:2013 [8], e verificar se há correlação entre a velocidade encontrada e a resistência obtida nos ensaios de compressão já realizados em laboratório de inspeção em obras de construção civil, denominado Beton \& Freitas Dantas.

Complementando ainda sobre fatores que contribuem com a durabilidade de uma construção, o cobrimento é um fator de suma importância. Segundo descrito na ABNT NBR 6118:2014 [9], a durabilidade da estrutura é altamente dependente das características do concreto, da espessura e qualidade do concreto do cobrimento da armadura. A espessura http: / / dx.doi.org/10.25286/repa.v2i3.682 
desta capa de concreto é importante para garantir a proteção das armaduras frente aos agentes agressivos, dependendo das condições de exposição deste concreto. [6]

Assim, além do ensaio não destrutivo com o ultrassom, foi realizado o mapeamento de pilares e vigas com o aparelho (pacômetro) que detecta a localização das armaduras, diâmetro e a espessura de cobrimento de concreto, para verificar se os cobrimentos executados estão de acordo com as prescrições da ABNT NBR 6118:2014 [9]. Viabilizando assim um controle de qualidade expedito para os profissionais da área que poderão monitorar a qualidade da estrutura já durante sua produção visando-se a vida útil de projeto (VUP) e durabilidade mínima de 50 (cinquenta) anos da estrutura do edifício objeto do presente estudo de caso.

Os ENDs foram realizados em um edifício residencial, em fase de acabamento, localizado no Bairro Jardins, na cidade de Aracaju, no Estado de Sergipe. O empreendimento é composto por 42 (quarenta e duas) unidades habitacionais, apresenta área total construída de 11.519,91 m², distribuídos em 01 pavimento térreo, 01 pavimento superior, 01 pavimento playground, 13 pavimentos tipos ( 10 ao $13^{\circ}$ ) e 01 ático (reservatório, barrilete e cobertura), sua tipologia estrutural é de laje maciça, com vigas e pilares, e o concreto com resistência característica (fck) de $30 \mathrm{MPa}$ e cobrimento das armaduras de 2,5 centímetros.

\section{Materiais e Métodos}

Para a realização dos ensaios, foram feitos procedimentos preliminares como a regularização da superfície dos elementos via lixamento manual e a calibração diária do equipamento de ultrassom através de barra acrílica referencial que acompanha o instrumento. Nas medições, inclusive calibrações, utilizou-se de gel apropriado com o fito de garantir um bom acoplamento entre o concreto e os transdutores.

As medições foram realizadas em todos os pilares e vigas de livre acesso localizados no primeiro pavimento superior do edifício, em face de que representa um dos pavimentos mais solicitados em termos de carregamentos.

Os ensaios foram executados conforme procedimentos da ABNT NBR 8802:2013 [8]. O equipamento de ultrassom utilizado foi da marca
Uslab com um par de transdutores planos de $54 \mathrm{KHz}$ e um detector de armadura (pacômetro) da marca Proceq. Para não haver influências das armaduras nas leituras ultrassonoras, realizava-se anteriormente, via pacometria, a localização das armaduras. O posicionamento dos transdutores do ultrassom foi sempre nos centros das malhas definidas pela pacometria. Esse pacômetro foi também utilizado para o mapeamento dos cobrimentos das armaduras nos pilares e vigas.

Dessa forma, depois de identificados os locais em que se encontravam as armaduras, tanto nos pilares quanto nas vigas, e devido ao bom acesso aos elementos estruturais, os transdutores foram posicionados no elemento estrutural de forma direta, como mostrado na Figura 1. Procediam-se 10 (dez) medições ultrassonoras para cada um dos 3 (três) pontos nos pilares, sendo, respectivamente, 30 (trinta) centímetros acima do piso, no meio do pilar e 30 centímetros abaixo da viga, totalizando-se 30 medições em cada pilar, bem como uma quantidade variada de pontos nas vigas pelo fato de haver algumas de pequeno comprimento longitudinal e/ou de acesso restrito a menos de 3 pontos. A leitura das velocidades nas ondas ultrassônicas foi feita diretamente no visor do equipamento em metros por segundo $(\mathrm{m} / \mathrm{s})$.

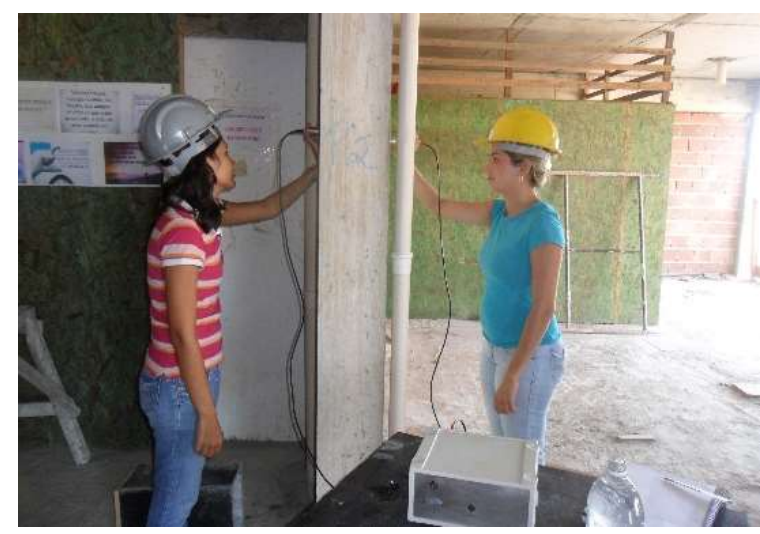

Figura 1: Ensaio de ultrassom, com os transdutores posicionados de forma direta.

Fonte: Autores (2017).

Para o cobrimento, depois de identificados os posicionamentos das armaduras no elemento estrutural, registrava-se o diâmetro da barra, descrito em projeto, no pacômetro e anotavam-se os valores encontrados dos cobrimentos a cada 50 (cinquenta) centímetros da extensão do elemento estrutural 
analisado e em todas as 4 (quatro) faces dos pilares e em 3 (três) das vigas. O ensaio está salientado na Figura 2.

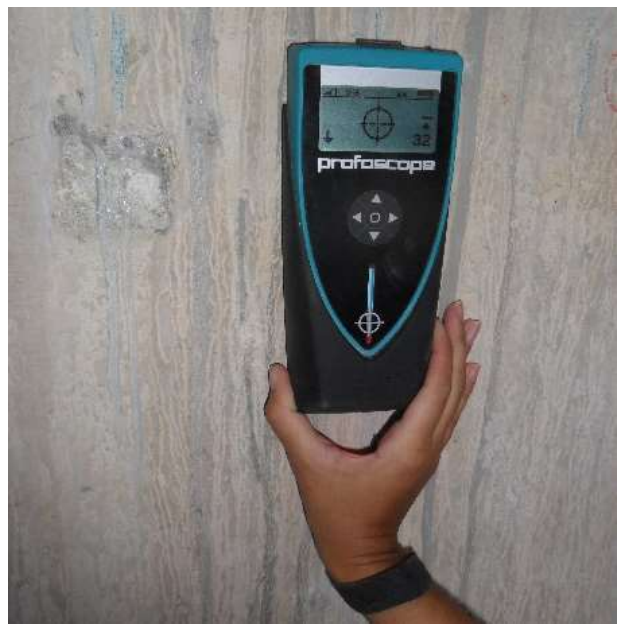

Figura 2: Localização da armadura no concreto e seu cobrimento por meio de pacometria.

Fonte: Autores (2017).

Após obtenção dos dados, realizou-se uma análise por meio do Software OriginPro 8.0 bem como a utilização da expressão matemática do coeficiente de Pearson, com o objetivo de verificar a existência de correlação aceitável estatisticamente entre as velocidades ultrassonoras medidas e as resistências laboratoriais aos 28 dias de ruptura à compressão. Os resultados e análises estão descritos a seguir.

\section{Resultados e Discussões}

Foram realizados inicialmente ensaios de ultrassom em 41 (quarenta e um) pilares e 20 (vinte) vigas para a análise da qualidade do concreto. Foram verificadas médias dos resultados obtidos de cada pilar e viga em comparação com as resistências mecânicas fornecidas pelo Laboratório da Beton, aos 28 dias de moldados, realizadas em amostras retiradas de cada caminhão betoneira que transportava os concretos no estado fresco até determinada área da obra a ser concretada, referentes aos pilares e vigas, conforme mostram as Tabelas $1 \mathrm{e}$ 2 , respectivamente.

Vale ressaltar que trabalhar com valores médios requer muita cautela logo que diagnosticar resultados analisando apenas a média pode acarretar em interpretações físicas equivocadas. [3]
Segundo Bungey et al. (2006) [10], se houver um gráfico de calibração confiável juntamente com boas condições de ensaio é possível atingir o limite de confiança de $95 \%$ sobre uma previsão de resistência de $\pm 20 \%$ relativo a uma área localizada de interesse.

Tabela 1: Média de resultados obtidos nas medições com o ultrassom em pilares e o fck encontrado nos corpos de prova no ensaio de compressão.

\begin{tabular}{c|c|c}
\hline Pilares & $\begin{array}{c}\text { Médias de } \\
\text { velocidade de } \\
\text { propagação } \\
\text { linear }(\mathbf{m} / \mathbf{s})\end{array}$ & $\begin{array}{c}\text { Resistência (fck) do } \\
\text { concreto após 28 } \\
\text { dias de moldado } \\
\text { (MPa) }\end{array}$ \\
\hline Pilar 1 ao 13 & 3736,98 & 32,8 \\
\hline Pilar 14 ao 29 & 3790,60 & 34,3 \\
\hline Pilar 30 ao 41 & 3754,13 & 32 \\
\hline Pilar 59 ao 70 & 3834,57 & 33,6 \\
\hline Pilar 59A ao & 3667,55 & 32 \\
$72 \mathrm{~A}$ & &
\end{tabular}

Fonte: Autores (2017).

Tabela 2: Média de resultados obtidos nas medições com o ultrassom em vigas e o fck encontrado nos corpos de prova no ensaio de compressão.

\begin{tabular}{c|c|c}
\hline Vigas & $\begin{array}{c}\text { Médias de } \\
\text { velocidade de } \\
\text { propagação linear } \\
(\mathbf{m} / \mathbf{s})\end{array}$ & $\begin{array}{c}\text { Resistência (fck) do } \\
\text { concreto após 28 dias } \\
\text { de moldado (MPa) }\end{array}$ \\
\hline Viga 6 e 120 & 3603,265 & 36,4 \\
\hline Viga 10 a 18 & 3682,74 & 37,8 \\
\hline Viga 20 & 3766,23 & 38,2 \\
\hline Viga 25 à 33 & 3668,686 & 36,9 \\
\hline Viga 109 & 3684 & 36,8
\end{tabular}

Fonte: Autores (2017).

Segundo a ABNT NBR 7215:1996 [11], os resultados da resistência mecânica são considerados satisfatórios tendo em vista que são superiores aqueles apresentados na especificação do projeto inicial, 30 (trinta) MPa.

Em relação aos resultados dos ensaios de ultrassom, esses valores são considerados ótimos, pois a qualidade do concreto se encaixa dentro deste nível considerado por Cánovas [12] (1998, apud SAHUINCO, 2011 [13]), como descrito na Tabela 3, já que as velocidades estão entre 3500 e 4500 m/s.

http: / /dx.doi.org/10.25286/repa.v2i3.682 
Utilização de ensaios não destrutivos, ultrassom e pacômetro, como métodos de verificação da resistência e qualidade do concreto em um edifício em Aracaju - Sergipe

Tabela 3: Critério utilizado para a avaliação da qualidade do concreto.

\begin{tabular}{c|c}
\hline $\begin{array}{c}\text { Velocidade de propagação } \\
\text { linear }(\mathbf{m} / \mathbf{s})\end{array}$ & $\begin{array}{c}\text { Qualidade do } \\
\text { concreto }\end{array}$ \\
\hline $\mathrm{V}>4500$ & Excelente \\
\hline $3500<\mathrm{V}<4500$ & Ótimo \\
\hline $3000<\mathrm{V}<3500$ & Bom \\
\hline $2000<\mathrm{V}<300$ & Regular \\
\hline $\mathrm{V}<2000$ & Ruim
\end{tabular}

Fonte: CÁNOVAS (1998) [12] apud SAHUINCO (2011) [13].

Para verificar o coeficiente de correlação de Pearson, foi utilizada a Equação 1 a seguir:

$$
\mathrm{r}=\frac{n \times \sum X i \times Y i-\left(\sum X i\right) \times\left(\sum Y i\right)}{\sqrt{\left(n \times \sum X i^{2}-\left(\sum X i\right)^{2}\right) \times\left(n \times \sum Y i^{2}-\left(\sum Y i\right)^{2}\right)}}
$$

Com o sentido de facilitar o entendimento e os cálculos, nas Tabelas 4 e 5 são detalhados os valores das variáveis e operações matemáticas necessárias à utilização da equação citada anteriormente.

Tabela 4: Variáveis para cálculo do coeficiente de correlação de Pearson em pilares.

\begin{tabular}{c|c|c|c|c|c}
\hline Pilares & $\mathbf{X}$ & $\mathbf{Y}$ & $\mathbf{X}^{2}$ & $\mathbf{Y}^{2}$ & $\mathbf{X \times Y}$ \\
\hline $\begin{array}{c}\text { Pilar 1 ao } \\
13\end{array}$ & 3736,98 & 32,8 & 13965019,52 & 1075,84 & 122572,944 \\
\hline $\begin{array}{c}\text { Pilar 14 } \\
\text { ao 29 }\end{array}$ & 3790,60 & 34,3 & 14368648,36 & 1176,49 & 130017,580 \\
\hline $\begin{array}{c}\text { Pilar 30 } \\
\text { ao 41 }\end{array}$ & 3754,13 & 32 & 14093492,06 & 1024,00 & 120132,160 \\
\hline $\begin{array}{c}\text { Pilar 59 } \\
\text { ao 70 }\end{array}$ & 3834,57 & 33,6 & 14703927,08 & 1128,96 & 128841,552 \\
\hline $\begin{array}{c}\text { Pilar 59A } \\
\text { ao 72A }\end{array}$ & 3667,55 & 32 & 13450923,00 & 1024,00 & 117361,600 \\
\hline $\begin{array}{c}\text { SOMA } \\
\text { Fonte: }\end{array}$ & 18783,83 & 164,7 & 70582010,02 & 5429,29 & 618925,840 \\
\hline
\end{tabular}

Fonte: Autores (2017).

Tabela 5: Variáveis para cálculo do coeficiente de correlação de Pearson em vigas.

\begin{tabular}{l|l|l|l|l|l}
\hline Vigas & $\mathbf{X}$ & $\mathbf{Y}$ & $\mathbf{X}^{2}$ & $\mathbf{Y}^{2}$ & $\mathbf{X} \times \mathbf{Y}$ \\
\hline
\end{tabular}

\begin{tabular}{c|c|c|c|c|c}
\hline $\begin{array}{c}\text { Viga 6 e } \\
120\end{array}$ & 3603,265 & 36,4 & 12983518,66 & 1324,96 & 131158,846 \\
\hline $\begin{array}{c}\text { Viga 10 } \\
\text { a 18 }\end{array}$ & 3682,74 & 37,8 & 13562573,91 & 1428,84 & 139207,572 \\
\hline Viga 20 & 3766,23 & 38,2 & 14184488,41 & 1459,24 & 143869,986 \\
\hline $\begin{array}{c}\text { Viga 25 } \\
\text { à 33 }\end{array}$ & 3668,686 & 36,9 & 13459256,97 & 1361,61 & 135374,513 \\
\hline $\begin{array}{c}\text { Viga } \\
109\end{array}$ & 3684,0 & 36,8 & 13571856,00 & 1354,24 & 135571,200 \\
\hline SOMA & $\begin{array}{c}18404,92 \\
1\end{array}$ & 186,1 & 67761693,95 & 6928,89 & 685182,117
\end{tabular}

Fonte: Autores (2017).

Adotando as médias das velocidades das ondas ultrassônicas como variáveis $X$ e as resistências adquiridas pelos concretos após 28 dias de moldados como $\mathrm{Y}$, e sendo " $\mathrm{n}$ " o número de variáveis, no caso do presente artigo $\mathrm{n}=5$, obteve-se as seguintes correlações descritas pelas Equação 2 e Equação 3 das medições de pilares e vigas respectivamente, substituindo os valores das Tabelas 4 e 5 na Equação 1:

$$
r=\frac{5 \times 618925,84-(18783,83) \times(164,7)}{\sqrt{\left(5 \times 70582010,02-(18783,83)^{2}\right) \times\left(5 \times 5429,29-(164,7)^{2}\right)}}
$$

(2)

Sendo encontrada uma correlação de $r=0,741$.

$$
r=\frac{5 \times 685182,117-(18404,921) \times(186,1)}{\sqrt{\left(5 \times 67761693,95-(18404,921)^{2}\right) \times\left(5 \times 6928,89-(186,1)^{2}\right)}}
$$

Sendo encontrada uma correlação de $r=0,867$.

Para que seja interpretado o coeficiente de correlação encontrado, segundo Shimakura (2006) [14], é preciso saber que as principais propriedades do mesmo são:

- $\quad$ intervalo de variação está entre -1 e +1 .

- O coeficiente de correlação é uma medida adimensional, ou seja, independente das unidades de medida das variáveis $\mathrm{X}$ e $\mathrm{Y}$. 
- Quanto mais próximo de +1 for " $r$ ", maior o grau de associação positivo entre $X$ e $Y$, com $X$ e $Y$ variando na mesma direção.

- Quanto mais próximo de -1 for " $r$ ", maior o grau de associação negativo entre $X$ e $Y$, com $\mathrm{X}$ e $\mathrm{Y}$ variando em sentidos opostos.

- Quanto mais próximo de zero estiver " $r$ " menor será a correlação linear entre $X$ e $Y$. No qual, " $r$ " sendo igual a zero indica que não há associação entre as variáveis.

Tabela 6: Parâmetro para interpretação do resultado de correlação.

\begin{tabular}{c|c}
\hline Valor de $\mathbf{r}(+$ ou $\boldsymbol{-})$ & Interpretação \\
\hline 0,00 a 0,19 & Correlação bem fraca \\
\hline 0,20 a 0,39 & Correlação fraca \\
\hline 0,40 a 0,69 & Correlação moderada \\
\hline 0,70 a 0,89 & Correlação forte \\
\hline 0,90 a 1,00 & Correlação muito forte
\end{tabular}

Fonte: SHIMAKURA (2006) [14].

É notável que a correlação encontrada entre as velocidades medidas com o ultrassom e a resistência à compressão do concreto é satisfatória, visto que na Tabela 6, de acordo com os resultados obtidos anteriormente, é considerada uma correlação forte, próximo de +1 , indicando que as variáveis $X$ e $Y$ variam na mesma direção.

Cada ponto apresentado nos gráficos de dispersão das Figuras 3 e 4 representa a média dos resultados obtidos para cada intervalo de pilar e viga no caminhão concretado. É possível observar que os resultados de ultrassom dos elementos estruturais apresentam diferentes valores, mas com pouca variação, um intervalo entre 3603,265 a 3834,57 m/s. Enquanto que o intervalo apresentado para a resistência mecânica teve uma variação entre 32 a 38,2 MPa. Podendo ser observado também que as dispersões dos pontos estão seguindo a reta, mostrando que conforme for aumentando os valores das velocidades obtidas pelo ultrassom (US) maiores serão os valores das resistências à compressão $(f c k)$, salientando que há correlação positiva, tendendo a +1 .

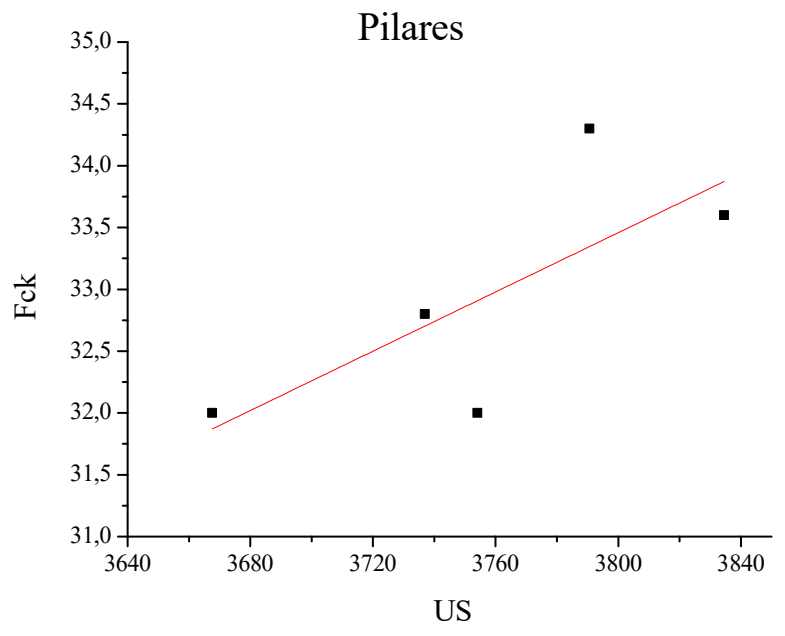

Figura 3: Gráfico de dispersão com valores médios do ensaio de ultrassom e da resistência mecânica dos pilares. Fonte: Autores (2017).

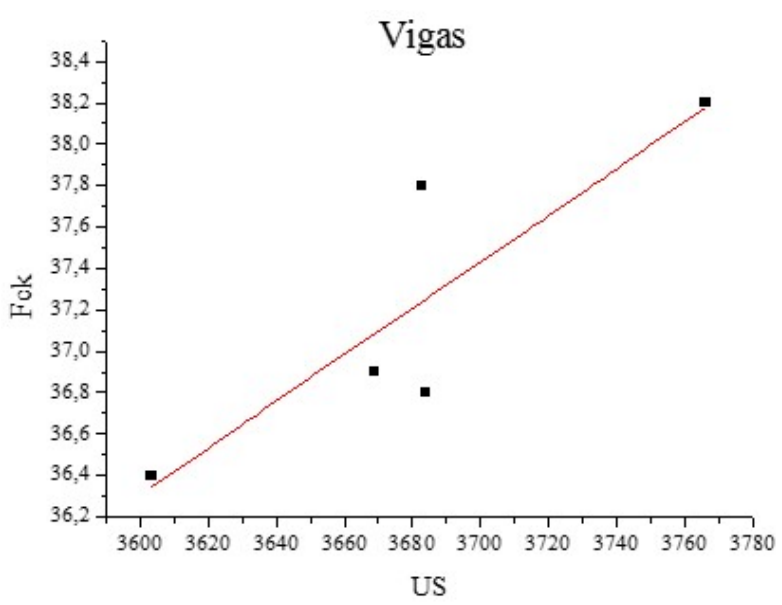

Figura 4: Gráfico de dispersão com valores médios do ensaio de ultrassom e da resistência mecânica das vigas. Fonte: Autores (2017).

Tratando-se do controle tecnológico, segundo Helene e Pacheco (2013) [1], toda estrutura de concreto, depois de acabada, possui uma série de características próprias que a diferencia daquela que foi especificada em projeto estrutural. Não havendo um perfeito controle e fiscalização durante a execução da obra, podem ser percebidas algumas dessas características, como exemplo as armaduras na estrutura não posicionadas como em projeto por alguma falha durante concretagem ou o não uso de espaçadores, o transporte, lançamento e adensamento inadequados gerando vazios, ninhos de

http: / / dx.doi.org/10.25286/repa.v2i3.682 
concretagem e/ou segregação no concreto, a cura pode ter sido insatisfatória formando fissuras por retração e não atingindo a resistência especificada, as fôrmas não têm as dimensões e resistência adequadas, os pilares não guardam o prumo nem o alinhamento (excentricidade) perfeito, a história de carregamento pode ter sido imprópria e certamente diferente daquela considerada no cálculo estrutural, etc.

O controle tecnológico de uma estrutura engloba a conferência de posição e bitola das armaduras, a geometria, o alinhamento, a estanqueidade e resistência das fôrmas, a qualidade dos materiais do traço, a eficiência da produção, as operações de transporte, lançamento e adensamento do concreto, o escoramento e a retirada do escoramento, o módulo de elasticidade, e outras variáveis de menor importância. [1]
Depois de realizados os ensaios de ultrassom em todos os elementos possíveis, foi utilizado o pacômetro para identificar se o cobrimento de concreto nas armaduras estava de acordo com o descrito em projeto, como uma das mais variadas formas possíveis de controle tecnológico da estrutura do edifício estudado. Foram encontrados pontos de cobrimento com 0,7 centímetros, considerado um valor muito baixo, até pontos com 6,8 centímetros, muito alto. Para melhor visualização, foram organizados esses cobrimentos nas Tabelas 7 e 8 apresentando apenas a média dos valores encontrados em cada face dos pilares e vigas, respectivamente.

A orientação Norte-sul, para melhor identificar o posicionamento das faces dos elementos estruturais estudados, foi tido como referencial Norte a fachada principal do edifício.

Tabela 7: Média do cobrimento de concreto nas armaduras longitudinais de pilares.

\begin{tabular}{c|c|c|c|c|c}
\hline Pilares & $\begin{array}{c}\text { Seção transversal } \\
(\mathbf{c m})\end{array}$ & $\begin{array}{c}\text { Face Norte } \\
\mathbf{( c m})\end{array}$ & $\begin{array}{c}\text { Face Sul } \\
(\mathbf{c m})\end{array}$ & $\begin{array}{c}\text { Face Leste } \\
(\mathbf{c m})\end{array}$ & $\begin{array}{c}\text { Face Oeste } \\
(\mathbf{c m})\end{array}$ \\
\hline P33 & $60 \times 20$ & 4,533 & 3,586 & 3,908 & 3,283 \\
\hline P25 & $115 \times 20$ & 3,799 & 3,149 & 4,170 & 4089 \\
\hline P15 & $115 \times 20$ & 3,358 & 3,717 & 4,042 & 4,282 \\
\hline P10 & $100 \times 20$ & 4,485 & 3,692 & 3,266 & 3,415 \\
\hline P18 & $100 \times 20$ & 4,033 & 3,449 & 4,019 & 4,019 \\
\hline P24 & $75 \times 20$ & 4,025 & 2,916 & 3,603 & 4,425 \\
\hline P57 & $25 \times 25$ & 3,675 & 2,775 & 4,200 & 3,708 \\
\hline P66 & $25 \times 25$ & 3,558 & 2,658 & 4,633 & 2,942 \\
\hline P69 & $25 \times 25$ & 3,666 & 3,433 & 3,992 & 3,508 \\
\hline
\end{tabular}

Fonte: Autores (2017). 
Tabela 8: Média do cobrimento de concreto nas armaduras longitudinais de vigas.

\begin{tabular}{c|c|c|c|c|c|c}
\hline Vigas & $\begin{array}{c}\text { Medidas } \\
(\mathbf{c m})\end{array}$ & $\begin{array}{c}\text { Face Norte } \\
(\mathbf{c m})\end{array}$ & $\begin{array}{c}\text { Face Sul } \\
(\mathbf{c m})\end{array}$ & $\begin{array}{c}\text { Face Leste } \\
(\mathbf{c m})\end{array}$ & $\begin{array}{c}\text { Face Oeste } \\
(\mathbf{c m})\end{array}$ & $\begin{array}{c}\text { Face de } \\
\text { baixo }(\mathbf{c m})\end{array}$ \\
\hline $\begin{array}{c}\text { V28 entre P32 e } \\
\text { P36 }\end{array}$ & $315 \times 20$ & 2,700 & 4,667 & - & - & 2,483 \\
\hline $\begin{array}{c}\text { V32 entre P33 e } \\
\text { P37 }\end{array}$ & $255 \times 20$ & 3,433 & 3,767 & - & - & 2,300 \\
\hline $\begin{array}{c}\text { V30 entre P24 e } \\
\text { P32 }\end{array}$ & $485 \times 15$ & 4,740 & 3,360 & - & - & 2,670 \\
\hline $\begin{array}{c}\text { V32 entre P25 e } \\
\text { P33 }\end{array}$ & $370 \times 15$ & 3,620 & 4,260 & - & - & 2,870 \\
\hline $\begin{array}{c}\text { V18 entre P33 e } \\
\text { P34 }\end{array}$ & $575 \times 15$ & - & - & 2,520 & 4,420 & 1,850 \\
\hline $\begin{array}{c}\text { V15 entre P23 e } \\
\text { P24 }\end{array}$ & $220 \times 15$ & - & - & 4,400 & 2,067 & 2,383 \\
\hline $\begin{array}{c}\text { V25 entre P23 e } \\
\text { P31 }\end{array}$ & $490 \times 15$ & 4,140 & 3,440 & - & - & 2,020 \\
\hline $\begin{array}{c}\text { V109 entre P66 } \\
\text { e P76 }\end{array}$ & $695 \times 20$ & - & - & 3,786 & 3,286 & 2,928 \\
\hline $\begin{array}{c}\text { V120 entre P66 } \\
\text { e P69 }\end{array}$ & $190 \times 20$ & 4,840 & 2,520 & - & - & 2,120 \\
\hline $\begin{array}{c}\text { V27 entre P23 e } \\
\text { P17 }\end{array}$ & $380 \times 15$ & 4,633 & 2,500 & - & - & 1,700 \\
\hline $\begin{array}{c}\text { V11 entre P18 e } \\
\text { P15 }\end{array}$ & $330 \times 15$ & - & - & 2,933 & 4,733 & 2,600
\end{tabular}

Fonte: Autores (2017).

Analisando as Tabelas anteriores, é perceptível que a grande maioria das vigas estudadas apresentava pelo menos uma das faces com cobrimento inferior ao mínimo indicado em projeto $(2,5 \mathrm{~cm})$, não ocorrendo o mesmo nos pilares, sendo que praticamente em sua totalidade apresenta uma média de cobrimento superior à mínima. Logo, o controle de execução durante a concretagem não foi seguido criteriosamente, havendo deslocamento da armadura durante a mesma.

\section{Conclusões}

Com os resultados desse estudo de caso, evidenciou-se que o monitoramento in situ da qualidade de produção e resistência mecânica do concreto é viável através dos ensaios não destrutivos de pacometria e ultrassonografia. Destarte, muito embora dependentes de outras variáveis que eventualmente poderiam influenciar na avaliação final precisa, possibilitam a coleta de informações qualitativas satisfatórias ao controle de qualidade das estruturas visando sua vida útil e durabilidade.
Salientou-se também a interessante portabilidade do uso dos ENDs nos canteiros de obras, pois consistem em métodos de ensaios rápidos, práticos, econômicos e qualitativos para o monitoramento da qualidade dos concretos estruturais durante sua execução e/ou já executados.

\section{Referências}

[1] HELENE, P.; PACHECO, J. Controle da Resistência do Concreto. Asociación Latinoamericana de Control de Calidad, Patologia y Recuperación de la Construcción - ALCONPAT Int., Mérida - México, 2013.

[2] EVANGELISTA, A. C. J. Avaliação da Resistência do Concreto Usando Diferentes Ensaios Não Destrutivos. Rio de Janeiro, 2002.

[3] CARVALHO, C. H. Estudo comparativo das técnicas não destrutivas de ultrassom e esclerometria na avaliação do desempenho mecânico de estruturas em concreto armado. 2013. 
[4] SCHIAVON, Karen Fernanda Bompan. Estudo da aplicação de ultrassom na medição de tensões em estruturas de concreto / Karen Fernanda Bompan Schiavon; orientador Vladimir Guilherme Haach. São Carlos, 2015.

[5] ANDREUCCI, RICARDO. Ensaio por ultrassom. ABENDI, Ed. Maio. 2014.

[6] GRANATO, J. E. Patologia das Construções. 2002.

[7] MACHADO M. D.; SHEHATA L. C. D.; SHEHATA E. M., Curvas de correlação para caracterizar concretos usados no Rio de Janeiro por meio de ensaios não destrutivos. IBRACON, Volume 2, 2009.

[8] Associação Brasileira de Normas Técnicas (ABNT). NBR 8802:2013 - Concreto endurecido Determinação da velocidade de propagação de onda ultrassônica.

[9] Associação Brasileira de Normas Técnicas (ABNT). NBR 6118:2014 - Projeto de estruturas de concreto - Procedimento.

[10] BUNGEY, J. H.; et al. Testing of concrete in structures. 4th Ed. New York, USA: Champan e Hall, 2006.

[11] Associação Brasileira de Normas Técnicas ABNT NBR 7215:1996. Determinação da resistência a compressão.

[12] CÁNOVAS, M.F. (1998). Patologia e Terapia do Concreto Armado. Editora PINI, São Paulo, 522 p.

[13] SAHUINCO, M. H. C. UTILIZAÇÃO DE MÉTODOS NÃO DESTRUTIVOS E SEMIDESTRUTIVOS NA AVALIAÇÃO DE PONTES DE CONCRETO. São Paulo, 2011.

[14] SHIMAKURA, S. E. Interpretação do coeficiente de correlação. LEG, UFPR, 2006. 